\title{
A rare case of laryngeal carcinoma in pregnancy Hui Mon Teh ${ }^{\mathrm{a}}$, Mohd Sayuti Razali ${ }^{\mathrm{b}}$
}

aDepartment of Otorhinolaryngology, Universiti Malaya, Kuala Lumpur, ' Department of Otorhinolaryngology, Universiti Sultan Zainal Abidin, Kuala Terengganu, Malaysia

Correspondence to Hui Mon Teh, MD, Department of Otorhinolaryngology, Pusat Perubatan Universiti Malaya, Lembah Pantai, Kuala Lumpur 59100, Malaysia. Tel: +60 177 274 819;

e-mail: huimon@hotmail.com

Received 26 June 2018

Accepted 16 July 2018

The Egyptian Journal of Otolaryngology 2018, 34:363-365

\begin{abstract}
Cancer in pregnancy is uncommon. The ethical problem of managing head and neck cancer in pregnancy has always been a common issue to ensure the mother and fetal well-being. Choices for the diagnostic and treatment modalities are crucial to ensure optimal outcome for the mother as well as to preserve fetal vitality. Best clinical decision for the pregnant patient should be made through a multidisciplinary approach. We are reporting a case of a 24-year-old female patient who presented with stridor owing to supraglottic squamous cell carcinoma at 13 weeks of gestation. She underwent tracheostomy as an emergency procedure and later had tumor excision using carbon dioxide laser at 15 weeks of gestation. She was subsequently decannulated off tracheostomy and later underwent normal vaginal delivery at term and had no recurrence within 2-year follow-up.
\end{abstract}

\section{Keywords:}

cancer, laryngeal carcinoma, pregnancy

Egypt J Otolaryngol 34:363-365

() 2018 The Egyptian Journal of Otolaryngology

$1012-5574$

\section{Introduction}

Laryngeal cancer in young age is extremely rare especially in pregnancy. In such circumstances, both maternal health and fetal well-being have to be taken into consideration to plan out the best treatment option that results in enhanced survival and less complications. With surgical intervention and closed postoperative surveillance, our patient had safe delivery without complication and no recurrence.

\section{Case history}

A 24-year-old woman at 13 weeks of gestation presented to the Emergency Department with an audible inspiratory stridor with only minimal of respiratory effort. She initially had a history of sore throat, fever, and cough for a month. Her symptoms became progressively worse with noisy breathing associated with hoarseness and odynophagia. Physical examination showed lungs were clear without ronchi. She was then put on oxygen support, intravenous antibiotic, and regular salbutamol nebulizers. Bedside flexible nasopharyngolaryngoscopy revealed a left supraglottic mass obstructing the supralaryngeal airway. MRI of the neck revealed a vague left supraglottic mass obstructing the upper airway with no enlarged cervical lymph node.

She underwent tracheostomy, and direct laryngoscopy showed a fungating mass at the left aryepiglottic fold obstructing the laryngeal inlet acting like 'ball and valve effect' and normal vocal cords. Histopathological tissue examination showed squamous cell carcinoma.

At 15 weeks of gestation, a complete excision of the tumor using carbon dioxide laser was performed.
Patient was successfully decannulated from tracheostomy tube later. She then subsequently underwent normal vaginal delivery and was blessed with a healthy baby boy at term. Subsequent followup for 2 years showed no recurrence (Figs 1-4).

\section{Discussion}

Cancer in pregnancy is extremely rare [1,2]. As women in developed societies today defer childbearing age to their third or fourth decades and the incidence of most cancers rise parallel to increasing age, the infrequent coincidence of cancer and pregnancy has become more common. A reported study in Denmark has shown that the incidence rate of cancer diagnosed during pregnancy ranges from 17 to 38 cases/100 000 births [3]. The reported seven frequently diagnosed cancers during pregnancy include breast cancer, cervical cancer, ovarian cancer, Hodgkin lymphoma, non-Hodgkin lymphoma, leukemia, and melanoma [4]. Therefore, our reported case of laryngeal tumor is considerably extremely rare not only among the young age group and particularly in pregnancy. In all life-threatening pregnancy cases, priority is always given to the maternal health. Owing to cancer, management in pregnancy carries detrimental effects to the mother and the fetus; therefore, the decisions and treatments itself will become more complicated.

This is an open access journal, and articles are distributed under the terms of the Creative Commons Attribution-NonCommercial-ShareAlike 4.0 License, which allows others to remix, tweak, and build upon the work non-commercially, as long as appropriate credit is given and the new creations are licensed under the identical terms. 
Figure 1

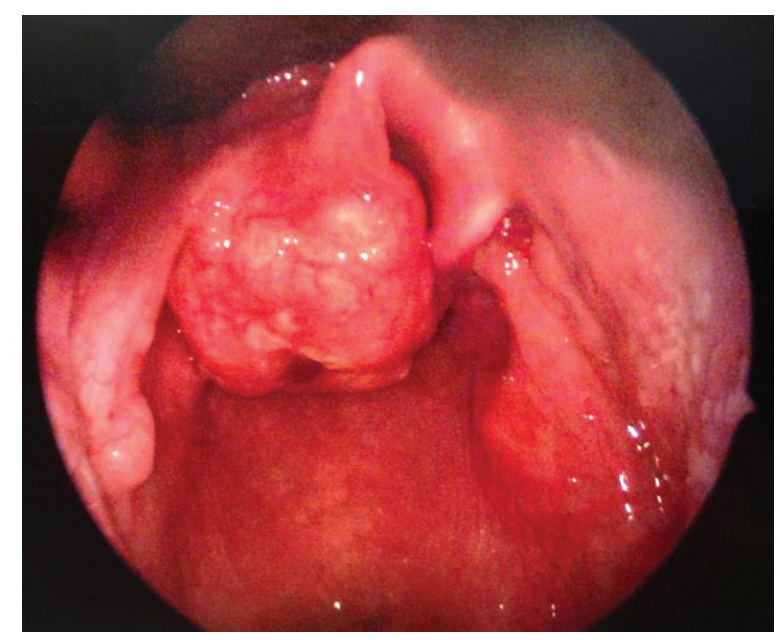

Direct laryngoscopy view of laryngeal mass.

\section{Figure 2}

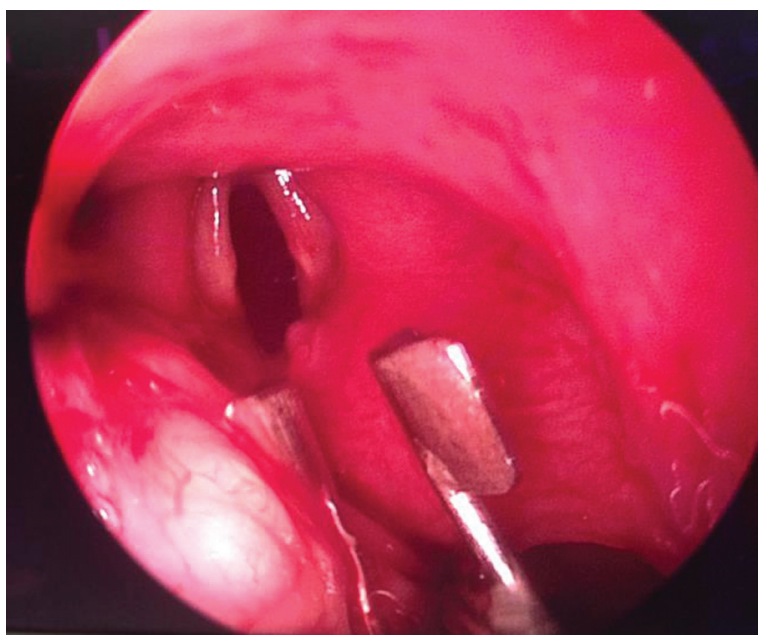

Laryngeal inlet with the mass.

With the evolution of imaging system, a simple bedside flexible nasolaryngoscopy is essential to look for the cause of upper airway obstruction without the need of sedation. Moreover, owing to the nonharmful effect of MRI to both mother and fetal well-being, MRI can be safely carried out. These two imaging systems reduce the necessity of ionizing radiation exposure produced by common imaging modalities such as radiography and computed tomography. However, every imaging modality has its own strengths and weaknesses. In this case, the laryngeal mass appeared as a mobile mass that affected the airway patency, which exhibited as an artefact image in the neck MRI.

Chemotherapy and radiotherapy both notably result in teratogenicity in the first trimester. The safety of the administration of chemotherapy drugs is always a questionable cue. Detrimental consequences of cytotoxic
Figure 3

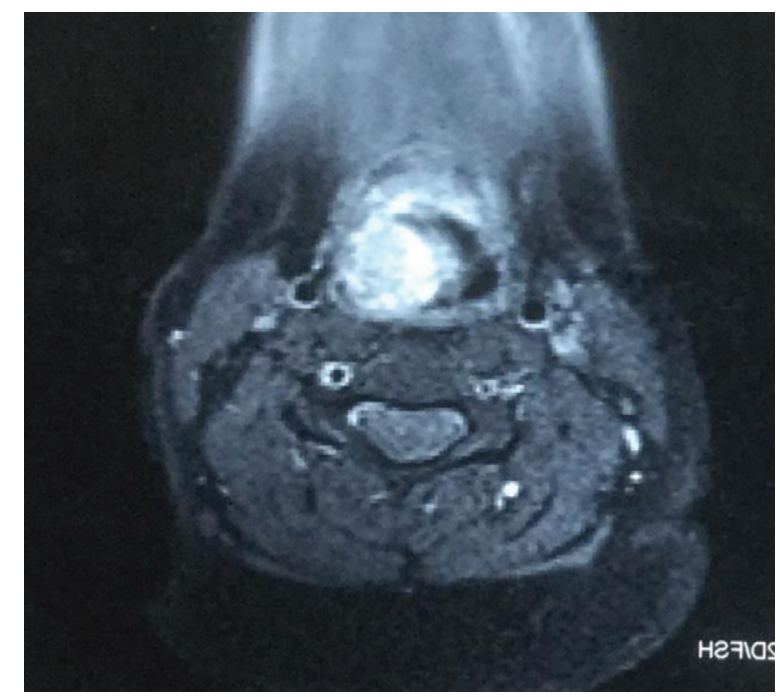

Axial view of MRI of the neck.

\section{Figure 4}

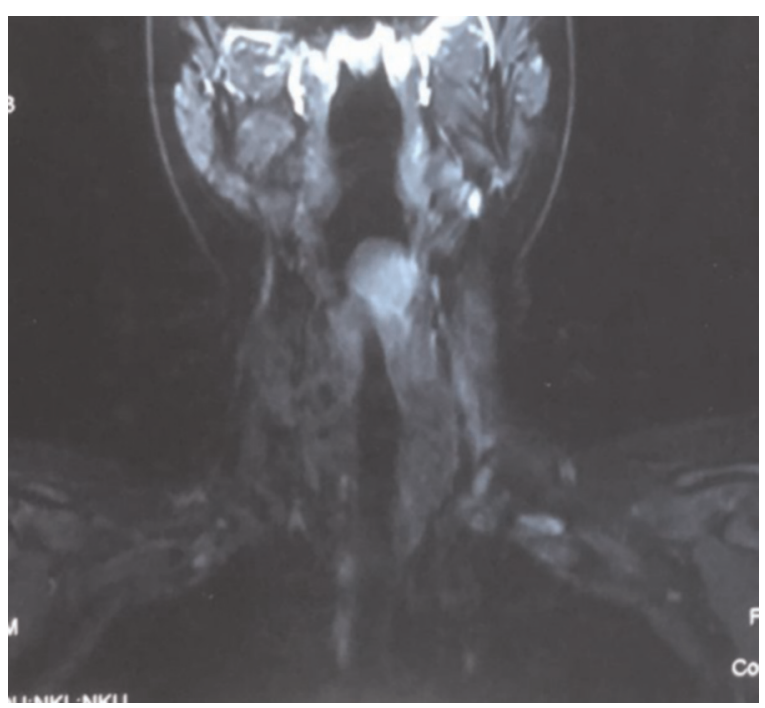

Coronal view of MRI of the neck.

drugs to the fetus or new-born include malformation, teratogenesis, mutations, carcinogenesis, organs toxicity, and growth retardation. For the mother, these drugs may result in abortion or sterility. Toxic effects were less reported when chemotherapy drugs were administrated in later trimesters comparatively to first trimester during embryogenesis. In addition, the crucial physiological changes during pregnancy potentially lead to significant change in anticancer drug pharmacokinetic properties [5]. Traditionally, prudent judgment is needed to minimize radiation exposure to a pregnant woman. There is always a concern of the risk of radiotherapy on the fetal well-being. Malignancies excluding the pelvis and abdomen have been reported to be treated with radiotherapy [6]. However, the circumstances vary depending on type 
and staging of cancer as well as the period of pregnancy. If radiotherapy is the best option during pregnancy, precautions need to be carefully planned out to reduce the fetal dose to the minimal but effective level.

Among all curative modalities on a solitary neoplastic tumor, surgical intervention is always a better choice. Maternal mortality is almost negligible with the advancement of the current surgical techniques and anesthesia, but the risk of miscarriage increases in the first trimester. Hence, surgery should be deferred as much as possible for the risk to the fetus to be minimal [7]. All pregnant women need to be evaluated preoperatively in the same manner as nonpregnant patients. Urgently needed surgery, as in our case which involved the airways, should be performed regardless of the trimester. Other reasons of performing the surgery early is to prevent the possibility of spread of tumor and metastasis. By using the carbon dioxide laser procedure, the surgery underwent smoothly, with minimal bleeding; accurate cutting was done with the help of a microscope, as it improves visualization of tumor and its margin. It also helps to preserve the normal tissue which is very important to maintain the integrity of the remaining airway. There is recommendation of performing surgery in the second trimester rather than the third trimester for the mechanical factors of the huge uterus and the risk of preterm labor being higher in the third trimester [8].

\section{Conclusion}

Management of pregnant women with cancer has changed in past few decades with the better knowledge of the physiology of pregnancy. Today, we are able to operate on a pregnant woman safely comparatively to the past, where termination of pregnancy might be the only best option for the patient. Nevertheless, preoperative evaluations must be carefully planned out to hinder the possible risks, and all maneuvers for the protection of fetus must be implemented. Multidisciplinary teams including family members should actively participate in the decision makings in such case to ensure optimal outcome of both mother health and fetal well-being.

\section{Financial support and sponsorship \\ Nil.}

\section{Conflicts of interest}

There are no conflicts of interest.

\section{References}

1 Bradley P, Raghavan U. Cancers presenting in the head and neck during pregnancy. Curr Opin Otolaryngol Head Neck Surg 2004; 12:76-81.

2 Yu Chow V, Wai Chan J, Man Ng R, Wei W. Management of head and neck tumours during pregnancy: case report and literature review. Asian J Surg 2008; 31:199-203.

3 Eibye S, Kjær S, Mellemkjær L. Incidence of pregnancy-associated cancer in Denmark, 1977-2006. Obstet Gynecol 2013; 122:608-617.

4 Stensheim H, Møller B, van Dijk T, Fosså S. Cause-specific survival for women diagnosed with cancer during pregnancy or lactation: a registrybased cohort study. J Clin Oncol 2009; 27:45-51.

5 Pavlidis N. Coexistence of pregnancy and malignancy. Oncologist 2002; 7:573-573.

6 Azim H Jr. Managing cancer during pregnancy. 2016.

7 Basta $\mathrm{P}$, Bak A, Roszkowski K. Cancer treatment in pregnant women. Contemp Oncol (Pozn) 2015; 19:354-360.

8 Fatum M, Rojansky N. Laparoscopic surgery during pregnancy. Obstet Gynecol Surv 2001; 56:50-59. 